\title{
Study of Gemcitabine Plus Nab-Paclitaxel-Based Chemotherapy Regimen as First-Line Treatment in Metastatic Pancreatic Carcinoma
}

\author{
Kshitij Domadia ${ }^{1}$ Varun Goel ${ }^{1}$ Venkata Pradeep Babu Koyyala ${ }^{1}$ Nivedita Patnaik ${ }^{2}$ \\ Krushna Chaudhari $^{1}$ Shubhra Raina ${ }^{1}$ D. C. Doval ${ }^{1}$ Vineet Talwar ${ }^{10}$
}

${ }^{1}$ Department of Medical Oncology, Rajiv Gandhi Cancer Institute and Research Centre, New Delhi, India; ${ }^{2}$ Department of Pathology, Rajiv Gandhi Cancer Institute and Research Centre, New Delhi, India

Address for correspondence Varun Goel, MBBS, MD, DNB, Department of Medical Oncology, Rajiv Gandhi Cancer Institute and Research Centre, Sector 5, Rohini, New Delhi 110085, India

South Asian J Cancer 2022;11(1):31-35. (e-mail: goelvarundoc@gmail.com).

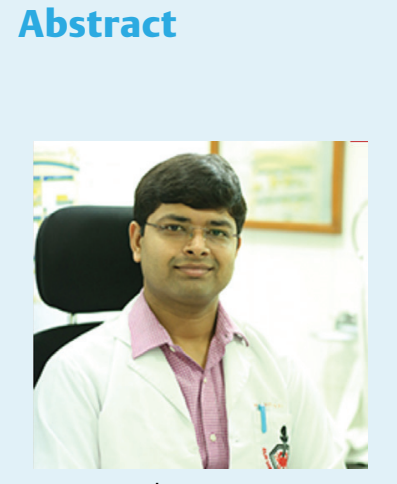

Varun Goel
Keywords

- pancreatic carcinoma

- gemcitabine

- nab-paclitaxel
Background The purpose of this study was to study the effectiveness of gemcitabine and nab-paclitaxel combination as first-line chemotherapy regimen for the treatment of metastatic pancreatic cancer. There is scarcity of data regarding efficacy and toxicity profile of this combination in Indian population.

Aims and Objectives The primary aim of this study was to assess efficacy of this regimen, for which evaluation done in terms of the objective response rate, progression-free survival (PFS), and overall survival. Safety data were also evaluated.

Materials and Methods In this prospective study, gemcitabine plus nab-paclitaxel combination chemotherapy was given as first line in metastatic pancreatic carcinoma patients till progression or appearance of grade $3 / 4$ toxicities with treatment.

Results The study was performed in 30 patients comprising 18 (60\%) males and 12 (40\%) females. The median age was 60 years. Median number of cycles administered were six cycles per patient. Seventeen patients (56.67\%) had a partial response and $0 \%$ had complete response. A total of seven (23.3\%) patients progressed on chemotherapy and six (20\%) had stable disease (SD). The disease control rate (responses and SD) was 76.7\%. The median PFS was 5.75 months. There was no statistically significant difference in terms of response rates and baseline CA 19-9 levels. Most common toxicities were hematological toxicities with rates of grade 3/4 anemia and neutropenia of $20 \%$. Among nonhematological toxicities, nausea $(46.67 \%)$ and fatigue $(30 \%)$ were the commonest.

Conclusion Combination of gemcitabine and nab-paclitaxel is active and well tolerated in advanced pancreatic carcinoma. To the best of our knowledge, this is the first such study conducted in India.
DOI https://doi.org/10.1055/s-0041-1735956 ISSN 2278-330X

How to cite this article: Domadia K, Goel V, Koyyala VPB et al. Study of Gemcitabine Plus Nab-Paclitaxel-Based Chemotherapy Regimen as First-Line Treatment in Metastatic Pancreatic Carcinoma South Asian J Cancer 2022;11(1):31-35.
(C) 2021. MedIntel Services Pvt Ltd. All rights reserved.

This is an open access article published by Thieme under the terms of the Creative Commons Attribution-NonDerivative-NonCommercial-License, permitting copying and reproduction so long as the original work is given appropriate credit. Contents may not be used for commercial purposes, or adapted, remixed, transformed or built upon. (https://creativecommons.org/licenses/by-nc-nd/ 4.0/)

Thieme Medical and Scientific Publishers Pvt. Ltd., A-12, 2nd Floor, Sector 2, Noida-201301 UP, India 


\section{Introduction}

Adenocarcinoma of pancreas is considered a malignancy having high mortality rates and poor prognosis. Despite significant advances in oncopharmacology, Disease-specific survival has not changed significantly in the past four decades, regardless of disease stage. It is considered curative in a very small subset of patients having localized disease.

According to the Surveillance, Epidemiology, and End Results (SEER) database, ${ }^{1}$ it is estimated that in 2020, there shall be 57,600 new cases of pancreatic carcinoma and an around 47,050 people will die of this disease. It is regarded as the 11th commonest cause of cancer in the United States and the third most common cause of cancer-related mortality highlighting the disproportionate mortality associated with it. Based on data from SEER 182009 to 2015, 5-year survival rate was $9.3 \%$ for pancreatic cancer. At diagnosis, most of pancreatic cancer is in advanced stage (53\% metastatic vs. $29 \%$ regional spread vs. $10 \%$ localized, and $8 \%$ unstaged). The incidence of pancreatic cancer in India is low (0.5-2.4 per 100,000 men and $0.2-1.8$ per 100,000 women). ${ }^{2}$

Early trials of chemotherapy for advanced pancreatic cancer were based on 5-fluorouracil (5-FU) and later, on 5FU combinations. Gemcitabine was compared with 5-FU/leucovorin in randomized trials in the 1990s and was approved as a first-line agent on the basis of a pivotal phase III trial. ${ }^{3}$ There were only few standard options for treating pancreatic carcinoma and they were limited to only gemcitabine until 2007, when the phase III results of gemcitabine plus erlotinib showed a 2-week overall survival advantage as compared with gemcitabine alone. ${ }^{4}$ Despite the relatively small magnitude of this survival benefit, this was the first agent, which had shown significant benefit in combination with gemcitabine in a phase III trial. FOLFIRINOX regimen was found to be superior to gemcitabine in terms of overall survival in a landmark phase 3 trial published in 2011, but the rates of hematological toxicity was significant. ${ }^{5} \mathrm{~A}$ breakthrough came in 2013 Metastatic Pancreatic Adenocarcinoma Clinical Trial (MPACT) study which showed improved survival with nab-paclitaxel plus gemcitabine combination therapy.

Most of these studies are from Western countries and there is a paucity of literature from the Indian subcontinent, so we decided to study the efficacy and toxicity profile of gemcitabine and nab-paclitaxel-based chemotherapy regimen in metastatic pancreatic carcinoma in Indian patients.

\section{Materials and Methods}

It was a prospective observational study. The primary aim of this study was to evaluate the objective response (complete response $[\mathrm{CR}] /$ partial response $[\mathrm{PR}] /$ stable disease $[\mathrm{SD}] /$ progressive disease) of gemcitabine and nab-paclitaxel-based chemotherapy regimen in metastatic carcinoma of pancreas and to evaluate the toxicity profile.

Thirty histologically or cytologically proven patients of metastatic pancreatic carcinoma at a tertiary cancer care center in North India were enrolled. Patients were enrolled from August 2018 to December 2019.

\section{Inclusion Criteria}

Eligible adults ( $\geq 18$ years) with histologically or cytologically proven metastatic adenocarcinoma of pancreas. Patients could have received 5-FU or gemcitabine as a radiation sensitizer in the adjuvant setting if the treatment had been received at least 6 months before starting on gemcitabine plus nab-paclitaxel. Eastern Cooperative Oncology Group (ECOG) performance status (PS) should be 0 , 1 , or 2. Patients should have adequate hematologic, renal, and hepatic function including a hemoglobin level of $\geq 9 \mathrm{~g} / \mathrm{dL}$, an absolute neutrophil count of $\geq 1.5 \times 109 / \mathrm{L}$, and a bilirubin level at or below the upper limit of the normal range.

\section{Exclusion Criteria}

The exclusion criteria are as follows: (1) ECOG PS 3 or 4. (2) Prior chemotherapy will not be permitted, except for 5-FU or gemcitabine given concurrently as a radiosensitizer. (3) Coexistent/synchronous malignancies. (4) Documented brain metastases (brain imaging not required in asymptomatic patients).

Approval from institutional scientific and ethical review boards had been taken.

\section{Treatment Protocol-Chemotherapy Regimen}

Gemcitabine $1,000 \mathrm{mg} / \mathrm{m}^{2} \mathrm{D} 1, \mathrm{D} 8, \mathrm{D} 15$ Q4 weekly and nabpaclitaxel $125 \mathrm{mg} / \mathrm{m}^{2} \mathrm{D} 1, \mathrm{D} 8, \mathrm{D} 15 \mathrm{Q} 4$ weekly until disease progression or unmanageable toxicity. Dose modification of chemotherapy was permitted according to protocol-specified criteria. Growth factor was not routinely given to all the patients.

Responses and progression were evaluated using Response Evaluation Criteria in Solid Tumors (RECIST version $1.1)^{6}$ after the completion of three cycles and then based on evaluation if it was decided to plan for three more cycles of same chemotherapy, then next re-evaluation after the completion of six cycles of chemotherapy, followed by every 3-monthly re-evaluation until disease progression or death.

The primary efficacy end point was progression-free survival (PFS) which was calculated from the start of therapy to the first observation of disease progression or death. Additional efficacy end points included the overall response rate (ORR) $(C R+P R)$ and disease control rate (DCR) $(\mathrm{CR}+\mathrm{PR}+\mathrm{SD})$.

Toxicity was assessed at every visit using the National Cancer Institute Common Toxicity Criteria version 4.0. Patients had complete blood cell counts evaluation along with kidney function test and liver function test before the start of each chemotherapy cycle. If patients had an absolute neutrophil count nadir $<500 / \mu \mathrm{L}$ (grade 4 neutropenia), the dose of chemotherapy was reduced by $25 \%$ in subsequent cycles. For platelet count nadirs $<50,000 / \mu \mathrm{L}$ along with bleeding complications, the dose of chemotherapy in subsequent cycles was reduced by $50 \%$.

\section{Statistical Analysis}

All analyses were performed with the statistical software SPSS version 23.0. Summary for continuous variable was presented in mean \pm standard deviation or median \pm interquartile range 
as per distribution of the data. Frequency and percentage were presented for categorical variable. For response and progression data, two-sided 95\% confidence intervals (CIs) were calculated based on an exact binomial probability at an $\alpha$ level of 0.05 . Continuous variables were compared using independent $t$-test or Mann-Whitney's test depending on the normality of the data and categorical variables were compared using chi-square test or Fisher's exact test. Survival analysis were estimated using the Kaplan-Meier's method or Cox's regression model wherever applicable. Statistical significance was defined as $p<0.05$.

\section{Results}

Patients were enrolled from August 2018 to December 2019 at a tertiary care cancer institute in India and the date of last follow-up was May 15, 2020. There were 72 histologically proven patients of pancreatic carcinoma who were found to be metastatic at presentation. Among them, 10 patients were not enrolled due to logistics, 8 patients did not meet inclusion criteria mostly due to poor PS. Six patients who had a good PS were managed with FOLFIRINOX. Fourteen patients were managed with other regimens such as single agent gemcitabine, gemcitabine plus erlotinib, and gemcitabine plus capecitabine. Thirty-four patients received gemcitabine + nab-paclitaxel-based combination therapy as a first-line treatment based on inclusion criteria mentioned earlier. Among them, three were lost to follow-up without a radiologic response evaluation and in one patient, the combination therapy was stopped after one cycle prior to any radiological evaluation due to grade 4 neurotoxicity. At the end of the study, 30 patients were available for final analysis.

In this present study, majority of cases, that is, $40 \%$ were in the 61 to 70 years age group. The mean age was 58.17 years (range, $34-75$ years). The median age was 60 years. The study was performed in 30 patients comprising 18 (60\%) males and 12 (40\%) females. In most cases, either two or more than two organs were involved and common sites of metastasis were liver, lymph nodes, omentum, and peritoneum. Seventeen patients (56.7\%) had liver metastasis, which was the most common site of metastasis. Six patients (20\%) had normal CA 19-9 levels.

A total of 173 cycles of treatment were administered with a median number of 6 cycles per patient (range, $2-12$ cycles). In this study, $33.3 \%$ of patients completed six cycles of chemotherapy. The major reason for discontinuation of chemotherapy was progression of disease. In one patient, chemotherapy had to be discontinued before response evaluation due to grade 4 neurotoxicity and this patient was not included in the analysis. Seven patients $(23 \%)$ in this study required the dose reduction at least in one cycle of chemotherapy due to toxicities. Fourteen (46.7\%) of patients in this study required delay in at least one cycle chemotherapy due to grades 3 and 4 hematologic toxicities or febrile neutropenia.

Hematological toxicities were the predominant toxicity noted with rates of grade $3 / 4$ anemia and neutropenia of $20 \%$. However, febrile neutropenia was seen in only one patient.

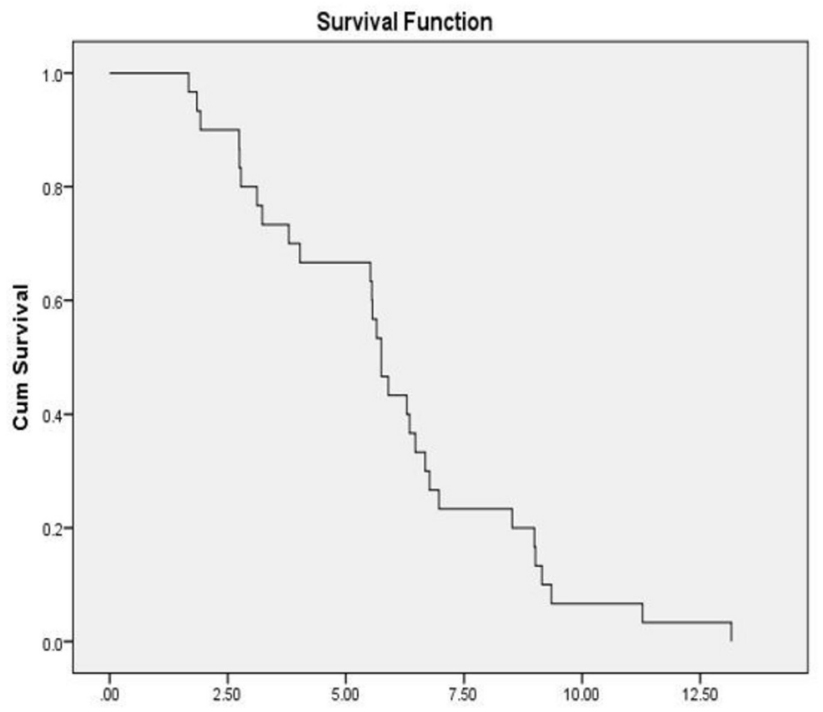

Fig. 1 Kaplan-Meier's distribution of progression-free survival.

Among nonhematological toxicities, nausea (46.67\%) and fatigue $(30 \%)$ were the commonest. Elevation in liver enzymes were seen in $13.33 \%$ of the patients. Seven patients (23.33\%) suffered from neuropathy, out of which four patients had grade 3 peripheral neuropathy.

The evaluation of response to chemotherapy was done by computed tomography (CT)/magnetic resonance imaging of the abdomen or positron emission tomography-CT if it was done as a staging procedure. In a total of 30 patients in this study, $56.67 \%(n=17)$ of patients had a PR and $0 \%$ had CR. A total of seven $(23.3 \%)$ patients progressed on chemotherapy and six (20\%) had SD. The DCR (responses and SD) was $76.7 \%$.

The median time to PFS was 5.75 months (range, 1.6713.16 months, 95\% CI: 4.82-6.95 months) (-Fig. 1). In subgroup analysis, five out of six patients having normal CA 19-9 levels at baseline responded having a response rate of $83.33 \%$. Response rates were 58.33 and $38.46 \%$ in subgroup of patients having CA $19-9<59$ upper limit of normal (ULN) and CA 19-9 >59 ULN. There was no statistically significant difference in terms of response rates and baseline CA 19-9 levels in this study $(p=0.239)$. Patients with normal, $<59$ ULN, and >59 ULN CA 19-9 levels had median PFS of 6.02, 5.75 , and 5.83 months, respectively $(p=0.908)$.

\section{Discussion}

Most patients diagnosed with pancreatic cancer either present with metastatic disease or develop it during the course of their illness. Currently available cytotoxic therapy provides modest survival benefits only. Few studies on gemcitabine and nab-paclitaxel-based chemotherapy in advanced pancreatic carcinoma have been reported from Western countries. Moreover, Indians are different from their Western counterparts, so the course of disease and response to different chemotherapeutic regimens may be different in an Indian scenario. The present study was prospectively designed to look at the efficacy, time to tumor progression, 
and tolerability of combination chemotherapy with gemcitabine and nab-paclitaxel-based regimes in 30 patients with advanced chemo naive pancreatic carcinoma who presented at a tertiary care cancer hospital of North India.

The median age at presentation in the present study was 60 years, which is similar to most of the published studies on the subject. The male (60\%) to female (40\%) ratio was $1.5: 1$, with PS 0 to 1 (86.67\%), 2 (13.33\%) which is also similar to reported literature. ${ }^{7}$ There was no statistically significant correlation seen between the age of the patient with the ECOG PS $(p=0.785)$.

The primary objectives of the present study were the ORR and PFS. In this study, CR was noted in $0 \%$ and PR in 56.7\% $(n=17)$ with ORR $=\mathrm{CR}+\mathrm{PR}$ of $56.7 \%$. The study also revealed SD in $20 \%(n=6)$ and $23.3 \%(n=7)$ progressed on chemotherapy. DCR $(C R+P R+S D)$ of the present study was $76.7 \%$. The median time to tumor progression in this study was 5.75 months (range, 0.36-11.52 months, 95\% CI: 4.64-7.08 months).

The response and outcome data were compared with landmark trial MPACT by Von Hoff et $\mathrm{al}^{7}$ and two other reported studies of Quinton et $\mathrm{al}^{8}$ and De Vita et al. ${ }^{9}$ These studies have shown DCR rates of 48,46 , and $70.7 \%$, respectively. DCR in the present study (76.7\%) was better than other reported studies with more number of patients achieving PR and less number of patients progressed on treatment. ORR of these studies were 23,19 , and $36.5 \%$, respectively. ORR of our study (56.7\%) was noted to be better than other reported studies. Difference in sample size as well as ethnic variations may have contributed to the difference in response rate noted across studies. The median PFS in these studies were 5.5, 4.4, and 6.7 months, respectively. Median PFS was comparable at 5.75 months in this present study. The response and the outcome (PFS) data of the present study were compared with these international studies (-Table 1 ).

In the present study, hematological toxicities were the predominant toxicity noted with rates of grade $3 / 4$ anemia and neutropenia of $20 \%$. However, febrile neutropenia was seen in only one patient. Majority of grade 3/4 side effects were hematological, and they were the commonest cause of

Table 1 Comparison of efficacy results in percentage

\begin{tabular}{|c|c|c|c|c|}
\hline $\begin{array}{l}\text { Response } \\
(\%)\end{array}$ & $\begin{array}{l}\text { Hoff } \\
\text { et al } \\
N=431\end{array}$ & $\begin{array}{l}\text { Quinton } \\
\text { et al } \\
N=74\end{array}$ & $\begin{array}{l}\text { De Vita } \\
\text { et al } \\
N=41\end{array}$ & $\begin{array}{l}\text { Present } \\
\text { study } \\
N=30\end{array}$ \\
\hline DCR & 48 & 46 & 70.7 & 76.7 \\
\hline CR & $<1$ & $<1$ & 4.8 & 0 \\
\hline PR & 23 & 18 & 31.7 & 56.7 \\
\hline PD & 20 & 16 & 29.3 & 23.3 \\
\hline SD & 27 & 27 & 34.2 & 20 \\
\hline PFS (mo) & 5.5 & 4.4 & 6.7 & 5.75 \\
\hline
\end{tabular}

Abbreviations: CR, complete response; DCR, disease control rate; PD, progressive disease; PFS, progression-free survival; $P R$, partial response $\mathrm{SD}$, stable disease. delay in chemotherapy cycle. Seven patients (23\%) required dose reduction in nab-paclitaxel and/or gemcitabine due to toxicities. Among nonhematological toxicities, nausea (46.67\%) and fatigue (30\%) were the commonest. Seven patients (23.33\%) suffered from neuropathy, out of which four patients had grade 3 peripheral neuropathy. Elevation in liver enzymes were seen in $13.33 \%$ of the patients. Thus, overall the treatment was well tolerated with no patient requiring treatment discontinuation due to toxicities. Chemotherapy toxicity data were compared with other studies done by Von Hoff et $\mathrm{al}^{7}{ }^{7}$ Quinton et $\mathrm{al}^{8}{ }^{8}$ and De Vita et al. ${ }^{9}$ On comparison with these studies, among grade 3/4 hematological toxicities, anemia was seen in more number of patients in the present study, while neutropenia and thrombocytopenia were seen in less number of patients. Among gemcitabine and nab-paclitaxel regimen, nab-paclitaxel is known to cause peripheral neuropathy with grade $3 / 4$ neurotoxicity reported as 17 and $12.2 \%$ in studies by Von Hoff et al ${ }^{7}$ and De Vita et al. ${ }^{9}$ In our study, four patients (13.33\%) had higher grades (3/4) of peripheral neuropathy. In one patient, treatment discontinuation was done prior to response evaluation due to grade 4 peripheral neuropathy and this patient was excluded from the final analysis.

There are very few published studies regarding outcomes of chemotherapy in patients with advanced pancreatic cancer in India. To the best of our knowledge, there is no published literature of Indian patients regarding the use of gemcitabine and nab-paclitaxel-based chemotherapy in advanced carcinoma pancreas.

Overall, the gemcitabine and nab-paclitaxel combination chemotherapy regimen was very well tolerated in the present study. The findings of the study have significant implications for clinical practice. Clinical outcome, toxicity profile of patients receiving chemotherapy for advanced pancreatic cancer has been observed to be similar to that reported from the west. The limitation of the present study was that it was a nonrandomized prospective observational study including small number of patients with short follow-up. Overall survival was not considered to be a part of this study as mentioned in the abstract. However, further long-term studies and randomized trials on Indians are warranted for confirmation. The combination of gemcitabine and nabpaclitaxel deserves further study to compare it with other standard regimens for pancreatic cancer especially FOLFIRINOX.

\section{Conclusion}

Combination of gemcitabine and nab-paclitaxel is active and well-tolerated chemo regimen in advanced pancreatic carcinoma. The response rate of $56.67 \%$ and the promising PFS are strong arguments for clinically using this combination in pancreatic carcinoma. To the best of our knowledge, this is the first such study conducted in India. More research is required, especially in the Indian subcontinent, to assess the efficacy and tolerability of this regimen in Indian patients. 
Financial Support

No financial support of any form has been taken from any agency for this study.

\section{Authors' Contribution}

Dr. Nivedita Patnaik did cytological and histopathological examination of the FNAC and biopsy. Dr. Varun Goel, Dr. Kshitij Domadia, Dr. Venkata Pradeep, Dr. Vineet Talwar, Dr. Shubhra Raina, Dr. Krushna Chaudhari, and Dr. DC Doval were involved actively in the clinical assessment and management of the patients. Dr. Varun Goel drafted the manuscript. All the others provided valuable inputs and guidance during the preparation of the manuscript. All authors read and approved the final manuscript.

Conflict of Interest

None declared.

\section{Acknowledgments}

We thank the Rajiv Gandhi Cancer Institute and Research Centre Hospital's staff, especially people working in the Department of Medical Oncology. We also thank the Action Cancer Hospital's staff, especially people working in the Department of Histopathology.

\section{References}

1 Surveillance Epidemiology and End Results (SEER) U.S. Cancer Statistics: Incidence and Mortality Report. Accessed
February 5, 2021 at: https://seer.cancer.gov/statfacts/html/ pancreas.html

2 Dhir V, Mohandas KM. Epidemiology of digestive tract cancers in India IV. Gall bladder and pancreas. Indian J Gastroenterol 1999; 18(01):24-28

3 Burris HA III, Moore MJ, Andersen J, et al. Improvements in survival and clinical benefit with gemcitabine as first-line therapy for patients with advanced pancreas cancer: a randomized trial. J Clin Oncol 1997;15(06):2403-2413

4 Moore MJ, Goldstein D, Hamm J, et al; National Cancer Institute of Canada Clinical Trials Group. Erlotinib plus gemcitabine compared with gemcitabine alone in patients with advanced pancreatic cancer: a phase III trial of the National Cancer Institute of Canada Clinical Trials Group. J Clin Oncol 2007;25 (15):1960-1966

5 Conroy T, Desseigne F, Ychou M, et al; Groupe Tumeurs Digestives of Unicancer PRODIGE Intergroup. FOLFIRINOX versus gemcitabine for metastatic pancreatic cancer. N Engl J Med 2011;364(19): 1817-1825

6 Eisenhauer EA, Therasse P, Bogaerts J, et al. New response evaluation criteria in solid tumours: revised RECIST guideline (version 1.1). Eur J Cancer 2009;45(02):228-247

7 Von Hoff DD, Ervin T, Arena FP, et al. Increased survival in pancreatic cancer with nab-paclitaxel plus gemcitabine. N Engl J Med 2013;369(18):1691-1703

8 Quinton AE, Gwynne SH, Yim KL. Nab-paclitaxel in combination with gemcitabine for the treatment of metastatic pancreas cancer: the South Wales experience. Med Oncol 2018;35(08): 115

9 De Vita F, Ventriglia J, Febbraro A, et al. Nab-paclitaxel and gemcitabine in metastatic pancreatic ductal adenocarcinoma (PDAC): from clinical trials to clinical practice. BMC Cancer 2016;16(01):709 